\title{
Reconstruction of Elasticity and Attenuation Maps in Shear Wave Imaging: An Inverse Approach
}

\author{
Armando Manduca ${ }^{1}$, Vinayak Dutt ${ }^{1}$, David T. Borup ${ }^{2}$, Raja Muthupillai ${ }^{1}$, \\ Richard L. Ehman ${ }^{1}$, and James F. Greenleaf ${ }^{1}$ \\ 1 Mayo Clinic and Foundation, Rochester MN 55905 \\ ${ }^{2}$ University of Utah, Salt Lake City UT 84112
}

\begin{abstract}
Acoustic shear waves of low frequency can be detected and measured using a phase contrast based magnetic resonance imaging technique called MR Elastography or phase measurement based ultrasound techniques. Spatio-temporal variations of displacements caused by the propagating waves can be used to estimate local values of the elasticity of the object being imaged. The currently employed technique for estimating the elasticity from the wave displacement maps, the local frequency estimator (LFE), has fundamental resolution limits and also has problems with shadowing and other refraction-related artifacts. These problems can be overcome with an inverse approach using Green's function integrals which directly solve the wave equation problem for the propagating wave. The complete measurements of wave displacements as a function of space and time over the object of interest obtained by the above techniques permit an iterative approach to inversion of the wave equation to obtain elasticity and attenuation maps.
\end{abstract}

\section{Introduction}

New techniques that can directly visualize propagating acoustic strain waves in tissue-like materials subjected to harmonic mechanical excitation have recently been developed: a phase contrast based magnetic resonance imaging technique called MR Elastography $[1,2]$ and a related ultrasound technique based on the phase of quadrature echo signals [3]. These techniques present the opportunity of generating medical images that depict tissue elasticity or stiffness. This is significant because palpation, a physical examination that assesses the stiffness of tissue, can be an effective method of detecting tumors, but is restricted to parts of the body that are accessible to the physician's hand.

The spatio-temporal variations of wave displacements measured by these techniques allow the calculation of local mechanical properties. In particular, estimation of the local wavelength (or frequency) of the shear wave propagation pattern at each point in the image allows one to quantitatively calculate local values of shear modulus across the image and generate an image of tissue elasticity. We have previously described a local frequency estimation (LFE) algorithm 
based on log-normal quadrature wavelet filters $[4,5]$ and have used it to calculate such images for synthetic data, test phantoms, excised tissue specimens, and in vivo [4]. These "elastograms" or "stiffness images" clearly depict areas of different elastic moduli in these objects, and calculated values for test phantoms correlate well with moduli calculated independently by mechanical means $[1,4]$. Although LFE is effective in reconstructing elasticity maps for simple objects, there are fundamental limits on the resolution it can achieve. The estimated values of the local frequency (and hence the shear modulus) are inaccurate within half a wavelength or so of a boundary with a different object. Also, LFE can produce artifacts in the presence of strong refraction and reflections. These limitations can be largely overcome by using an approach based directly on the wave equation, inverting the equation to estimate the mechanical properties of the object being imaged, as described below.

\section{Inverse Approach}

Any kind of wave propagation through an object has to satisfy the wave equation $[6,7]$,

$$
\rho(\bar{x}) \nabla \cdot\left\{\rho^{-1}(\bar{x}) \nabla \phi(\bar{x})\right\}+k^{2}(\bar{x}) \phi(\bar{x})=0
$$

where $\rho(\bar{x})$ is the medium density distribution, $k(\bar{x})$ is the complex wave number, $k^{2}(\bar{x})=\omega^{2}(\kappa(\bar{x})+j[\alpha(\bar{x}) / \omega)]$, where $\kappa(\bar{x})$ is medium compressibility and $\alpha(\bar{x})$ is the absorption factor, and $\phi(\bar{x})$ could be acoustic pressure or displacement. Since shear wave elastography provides information about $\phi(\bar{x})$ at every point $\bar{x}$ in the object, it should be possible to invert equation (1) to solve for the material properties $\rho(\bar{x}), \kappa(\bar{x})$ and $\alpha(\bar{x})$. This would provide the density, elasticity and attenuation maps for the object under investigation. We will normalize by density below and, from that point on, ignore density variations - in effect, assuming a uniform density of 1.0 everywhere (a fairly good approximation for soft tissues).

By a change of variable in equation (1), $\Phi=\phi / \sqrt{\rho}$, the equation can be written as the standard Helmholtz equation,

$$
\left[\nabla^{2}+\epsilon^{2}(\bar{x})\right] \Phi(\bar{x})=0
$$

where $\epsilon^{2}=k^{2}-\sqrt{\rho} \nabla^{2} \frac{1}{\sqrt{p}}$. This can be solved if the incident energy into the object is known (i.e., the wave equation solution in the absence of the object). The solution in terms of the Green's function can be written as the volume integral $[6,7]$,

$$
\Phi(\bar{x})=\Phi_{i}(\bar{x})-\epsilon_{0}^{2} \iiint_{V} \gamma\left(\bar{x}^{\prime}\right) \Phi(\bar{x}) g\left(\bar{x}-\bar{x}^{\prime}\right) d \bar{x}^{\prime}
$$

where $\epsilon_{0}$ is the value of $\epsilon$ for the background medium, $\gamma$ is relative value of $\epsilon^{2}$ normalized by $\epsilon_{0}^{2}, \gamma(\bar{x})=\left(\frac{\epsilon(\bar{x})}{\epsilon_{0}}\right)^{2}-1, g(\bar{x})$ is the Green's function of the wave and 
$\Phi i(\bar{x})$ is the incident wave distribution. Equation (3) can be used to devise an iterative method to solve for $\gamma$ (which we term the object function). The real part of $\gamma$ contains the elasticity information and the imaginary part the attenuation information. Such an estimator based on wave equation inversion should robustly handle situations with interference and refraction, which cause problems for local frequency estimation techniques, and should also provide higher resolution estimates of the mechanical properties.

If the functions $g(\bar{x})$ and $\Phi(\bar{x}) g(\bar{x})$ are written in terms of basis functions $\phi_{j}(\bar{x})$ which are shifted versions of each other, $\phi_{j}(\bar{x})=\phi\left(\bar{x}-\bar{x}_{j}\right)$,

$$
g(\bar{x})=\sum_{j} g\left(\bar{x}_{j}\right) \phi\left(\bar{x}-\bar{x}_{j}\right)
$$

and

$$
\gamma(\bar{x}) \Phi(\bar{x})=\sum_{j} \gamma\left(\bar{x}_{j}\right) \Phi\left(\bar{x}_{j}\right) \phi\left(\bar{x}-\bar{x}_{j}\right)
$$

then eq. (3) can be written as [6],

$$
\Phi(\bar{x})=\Phi_{i}(\bar{x})+\sum_{j} \gamma\left(\bar{x}_{j}\right) \Phi\left(\bar{x}_{j}\right) C\left(\bar{x}, \bar{x}_{j}\right)
$$

where

$$
C\left(\bar{x}, \bar{x}_{j}\right)=-\epsilon_{0}^{2} \iiint_{V} \phi\left(\bar{x}^{\prime}-\bar{x}_{j}\right) g\left(\bar{x}^{\prime}-\bar{x}\right) d \bar{x}^{\prime}
$$

The Green's function is a modified Bessel's function, $\frac{1}{4 i} H_{0}^{(2)}\left(k_{0} r\right)$, for the 2-D wave propagation problem and a complex sinusoid, $\frac{\exp \left(i k_{0} r\right)}{r}$, for the 3-D wave propagation problem. For a given sampling function, the basis function, $\phi(x)$, is known (e.g., sinc function for uniform sampling) and thus the coefficients, $C_{j}\left(\bar{x}, \bar{x}_{k}\right)$, of equation (7) can be estimated numerically.

If the field is measured at a finite set of grid points $\bar{x}_{k}$, then eqn. (6) can be written as a linear transformation,

$$
\Phi\left(\bar{x}_{k}\right)=\Phi_{i}\left(\bar{x}_{k}\right)+\sum_{j} \gamma\left(\bar{x}_{j}\right) \Phi\left(\bar{x}_{j}\right) C\left(\bar{x}_{k}, \bar{x}_{j}\right) .
$$

This equation can be iteratively solved for the total field, $\Phi(\bar{x})$, if the object function, $\gamma(\bar{x})$, and the incident field, $\Phi_{i}(\bar{x})$, are known. We have solved this forward problem by adapting a biconjugate gradient descent method previously shown to be useful for a related problem in diffraction tomography [8]. This allows the calculation of simulated data fields for known objects.

Conversely, if the total field, $\Phi(\bar{x})$, and the incident field, $\Phi_{i}(\bar{x})$, are known, then the inverse problem is to solve for the object function, $\gamma(\bar{x})$, obtaining the 
elasticity and attenuation values across the object. Equation (8) can be iteratively solved for $\gamma(\bar{x})$ using a conjugate gradient descent based pseudo inverse procedure based on modifications to the software described above [8].

Note that the total field at each point is a complex number, of which the measured displacement at a given moment is the real part. These complex displacements are measurable with both MR and ultrasound based shear wave elastography techniques by obtaining wave field images at different phases of the acoustic wave.

\section{Validation}

We first tested the efficacy of the inverse approach by simulating observed wave fields for given object functions using the forward scattering method described above. These wave fields were used to obtain estimates of the object function using the local frequency estimation algorithm. These estimates were then used as initial guesses in the iterative conjugate gradient inverse solutions for the object functions to evaluate the improvements in the reconstructed object functions over the LFE estimates. The inverse approach was also tested on in-vivo breast wave field images to verify the efficacy of the inverse approach for real data.

\subsection{Simulations}

Simple object functions with shear modulus and attenuation variations were used for simulating the wave field which would be measured in a shear wave imaging experiment, using the forward calculation method described above. The simulations used an image grid size of $128 \times 128$ with $1 \mathrm{~mm}$ pixel size. Simulations were performed for a shear wave frequency of $250 \mathrm{~Hz}$ with background wave speed of $2.56 \mathrm{~m} / \mathrm{s}$. Figure 1 shows the object function, the computed total field, and the reconstruction for one such experiment. This simulation does not have noise added.

The LFE was used to estimate the real part of the object function (LFE can not solve for the attenuation), using the real part of the field. The inverse approach then used this LFE estimate as its initial guess for the object function. From the figures it can be seen that the inverse approach shows improved edges and sharper object definitions than the LFE, as well as providing information on the attenuation (the imaginary component of the object function).

Simulations such as this show that the LFE has good reconstruction ability, but objects are blurry, edges are poorly defined, and some artifacts are obvious. The inverse approach significantly sharpens the reconstruction and reduces the artifacts. If the inverse approach is started from a random initial state instead of from the LFE estimate, the solutions are very similar, but more iterations are required. Typical computation speeds are 2-3 seconds for the LFE, 1 hour for the inverse approach starting from the LFE (200 iterations), and 75 minutes for the LFE from a random initial state (250 iterations). 


\subsection{Sensitivity to Incorrect Incident Field}

The inverse algorithm requires an incident field to be defined everywhere in the image - essentially, what the field would be if the entire image had an object function of unity and zero attenuation. This may be difficult to estimate in a real experimental situation. To check the sensitivity of the reconstruction to variability in the incident field specification, reconstruction was attempted with the incident field incorrectly specified to be of twice the proper amplitude and wavelength. The results were that the shear modulus (real part) was correctly reconstructed, but with an offset to the object function - that is, the background was estimated to have an object function of 3.0 instead of 1.0 , exactly what is needed to counter the effect of the incorrect input and yield the observed wavenumber. Similarly, the too-high amplitude specification was "corrected" by the algorithm by assigning a large attenuation to the pixels on the left edge of the image, which immediately brings the wave amplitude down to the proper value. This indicates that the algorithm is quite robust to inaccuracies in the amplitude and background wavenumber specification for the incident field.

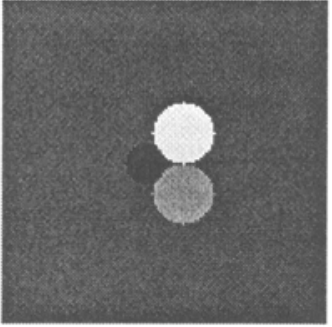

(a)

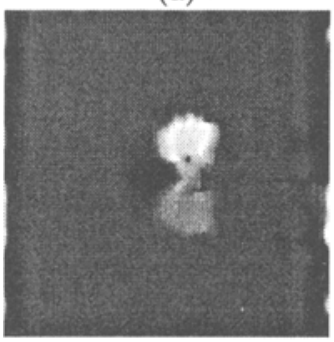

(d)

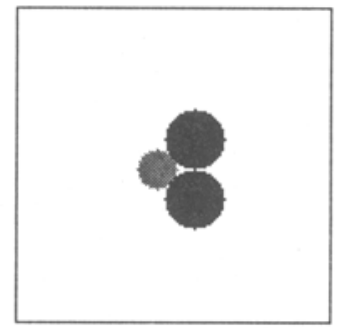

(b)

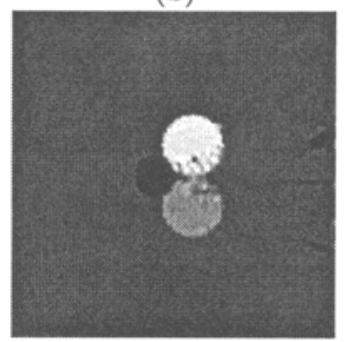

(e)

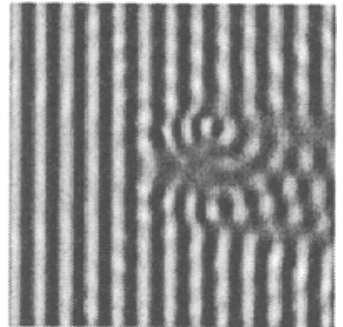

(c)

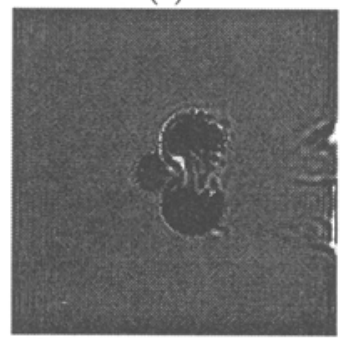

(f)

Fig. 1. Results for a simulated object function consisting of three circular objects with differing shear modulus and attenuation embedded in a uniform background. (a) and (b) show the real (shear modulus) and imaginary (attenuation) parts of the object function. (c) shows the real part of the complex total field, with the incident waves coming in from the left. (d) shows the LFE result. (e) and (f) show the real and imaginary parts of the object function using the inverse approach. 


\subsection{Sensitivity to Noise}

The sensitivity of the algorithm to noise in the data was studied by adding Gaussian noise of varying amplitude to noise-free simulations such as those above. Fig. 2(a) shows a complicated psi-shaped object function, which (with no attenuation) yields the total field shown in Fig. 2(b). The LFE does a surprisingly good job of reconstructing the object (Fig. 2(c)), but artifacts and blurriness are evident. The inverse approach yields a much sharper and more accurate reconstruction (Fig. 2(d)) in the noise-free case, although some artifacts are present. Results when noise is added are shown in Figs. 2(e-f). At the $1 \%$ level, the noise has a substantial effect on the reconstruction, and at the $4 \%$ level, the reconstruction is seriously compromised. The LFE results at these noise levels (not shown) differ little from the noise-free case. It is evident that the inverse algorithm as currently formulated is quite sensitive to noise in the data, and reducing this sensitivity by regularizing the algorithm in some way is a priority for future research.

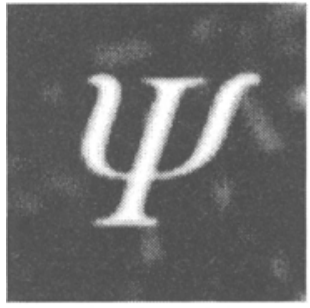

(a)

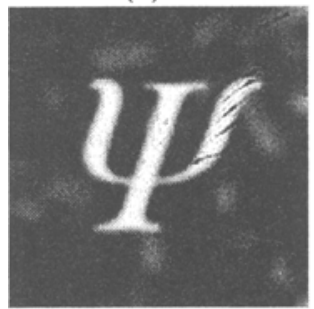

(d)

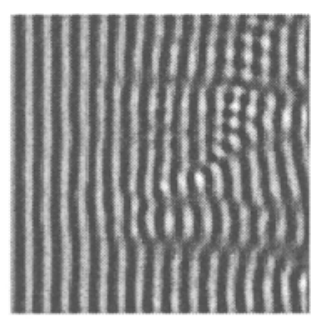

(b)

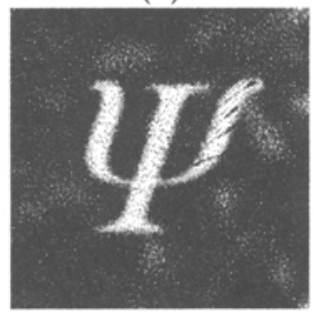

(e)

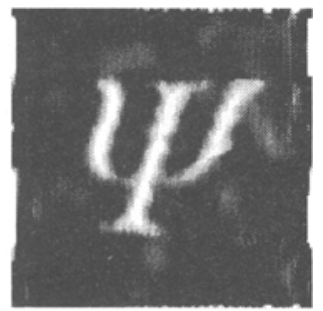

(c)

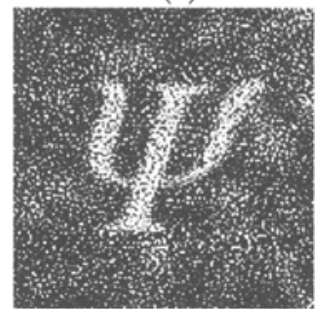

(f)

Fig. 2. Results for a simulation consisting of a psi-shaped object and smaller noise objects in a uniform background. (a) Object function: Real part of the object function. The imaginary part of the object function is zero (no attenuation). (b) The total field produced by such an object function (real part). (c) The LFE based reconstruction of the real part of the object function. (d) The real and part of the object function using the inverse approach. (e) The real and part of the object function using the inverse approach, after $1 \%$ Gaussian noise was added to the total field. (f) The real and part of the object function using the inverse approach, after $4 \%$ Gaussian noise was added to the total field. 


\section{Experimental Results}

Fig. 3 shows the results for shear wave measurements for an in-vivo MRE breast exam on a volunteer. The data was acquired at a shear wave frequency of 100 $\mathrm{Hz}$ on a $16 \mathrm{~cm}$ field of view on a $256 \times 256$ grid. The LFE reconstruction (not shown) is unable to distinguish fatty tissue from the glandular tissue (as seen in the magnitude image). Some structure and distinction between the two tissues is seen with the inverse approach, in both the elasticity and attenuation images (Fig. 3(b-c)). The inverse approach is thus able to provide estimates of two distinct quantities, shear modulus and shear wave attenuation, which can provide complementary information on tissue properties.

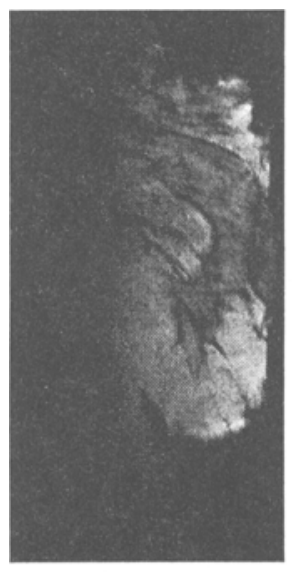

(a)

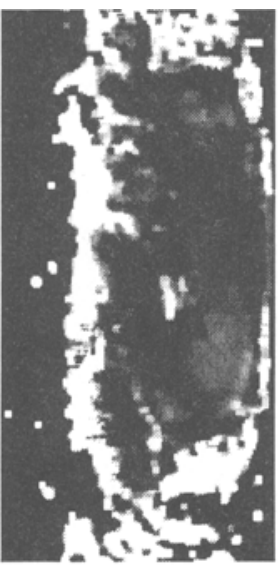

(b)

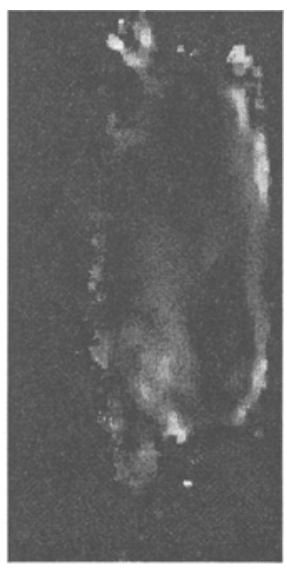

(c)

Fig. 3. Elasticity mapping for in-vivo breast MRE data. (a) The MR magnitude image of the scan. The breast rests on a vibrating surface at the right and the chest wall is to the left. (b) and (c) are the real (relative shear modulus) and the imaginary (attenuation) parts of the reconstructed object function using the inverse approach.

\section{Conclusion}

Acoustic shear wave elastography can provide the spatio-temporal distribution of shear wave propagation using either a phase contrast based MRI technique or an ultrasound based echo phase imaging technique. Such low frequency shear wave images can be utilized to reconstruct the elasticity and attenuation maps of the object imaged. The local frequency estimation (LFE) algorithm currently used provides useful reconstructions, but with limited resolution and some sensitivity to interference and refraction. The inverse approach to reconstruction described here provides sharper and more robust reconstructions, as demonstrated on simulations as well as on experiments with in-vivo breast images. 
The inverse approach also provides the attenuation maps, which are not obtainable with LFE. The inverse approach does show more sensitivity to noise than the LFE (not discussed here). Reducing this sensitivity to noise and speeding up the algorithm are priorities for future research. The inverse approach developed here can also be extended to 3-D quite simply via the use of the 3-D Green's function, although the computation times may become prohibitive.

\section{References}

1. R. Muthupillai, D. J. Lomas, P. J. Rossman, J. F. Greenleaf, A. Manduca, and R. L. Ehman, "Magnetic resonance elastography by direct visualization of propagating acoustic strain waves," Science, vol. 269, pp. 1854-1857, Sept. 1995.

2. R. Muthupillai, P. J. Rossman, D. J. Lomas, J. F. Greenleaf, S. J. Riederer, and R. L. Ehman, "Magnetic resonance imaging of transverse acoustic strain waves," Mag. Res. Med., vol. 36, pp. 266-274, 1996.

3. V. Dutt, R. R. Kinnick, and J. F. Greenleaf, "Shear wave displacement measurement using ultrasound," in IEEE Ultrasonics Symp. Proc., (New York), pp. 1185-1188, IEEE, 1996.

4. A. Manduca, R. Muthupillai, P. J. Rossman, J. F. Greenleaf, and R. L. Ehman, "Image processing for magnetic resonance elastography," Medical Imaging 1996: Image Processing, SPIE vol. 2710, pp. 616-623, 1996.

5. H. Knutsson, C.-F. Westin, and G. Granlund, "Local multiscale frequency and bandwidth estimation," in Proceedings of the 1994 IEEE International conference on Image Processing, (Los Alamitos, CA), pp. 36-40, IEEE Computer Society Press, 1994.

6. S. A. Johnson and M. K. Tracy, "Inverse scattering solutions by a sinc basis, multiple source, moment method - part I: Theory," Ultrasonic Imaging, vol. 5, no. 4, pp. 361$375,1984$.

7. S.-Y. Kim, H.-C. Choi, J.-M. Lee, and J.-W. Ra, "Inverse scattering scheme based on the moment method in the spectral domain, part I: Theory," Ultrasonic Imaging, vol. 14, pp. 16-28, Jan. 1992.

8. D. T. Borup, S. A. Johnson, W. W. Kim, and M. J. Berggren, "Nonperturbative diffraction tomography via gauss-newton iteration applied to the scattering integral equation," Ultrasonic Imaging, vol. 14, no. 1, pp. 69-85, 1992. 